ÉGYPTE monde arabe

\section{Égypte/Monde arabe}

15-16 | 1993

Les crises soudanaises des années 80

\title{
Le Front national islamique : idéologie et pratique
}

Idris Salim ElHassan

\section{(2) OpenEdition}

Journals

Édition électronique

URL : https://journals.openedition.org/ema/1101

DOI : 10.4000/ema.1101

ISSN : 2090-7273

Éditeur

CEDEJ - Centre d'études et de documentation économiques juridiques et sociales

Édition imprimée

Date de publication : 31 décembre 1993

Pagination : 227-247

ISSN : 1110-5097

\section{Référence électronique}

Idris Salim ElHassan, «Le Front national islamique : idéologie et pratique », Égypte/Monde arabe [En ligne], 15-16 | 1993, mis en ligne le 14 décembre 2016, consulté le 07 juillet 2022. URL : http:// journals.openedition.org/ema/1101; DOI : https://doi.org/10.4000/ema.1101

Ce document a été généré automatiquement le 7 juillet 2022.

Tous droits réservés 


\title{
Le Front national islamique : idéologie et pratique
}

\author{
Idris Salim ElHassan
}

\section{NOTE DE L'ÉDITEUR}

Texte traduit de l'arabe. Traduction revue et adaptée par François Ireton.

1 Les mouvements islamiques soudanais, qui connaissent actuellement une grande vitalité et qui; pour certains, ont construit les instruments de leur expression politique, sont à replacer dans le contexte général de renouveau islamique contemporain que connaît le monde, arabo-musulman, dont le Soudan fait, en tant qu'État, partie. Dans cet article, nous aborderons principalement les aspects politiques que présente ce renouveau au Soudan, en ne faisant allusion qu'en cas de nécessité à ses aspects non politiques et aux liens qu'entretiennent les mouvements islamiques soudanais avec ceux des autres pays musulmans. Plus précisément, nous nous centrerons sur le Front national islamique (FNI, issu des Frères musulmans) et nous nous en tiendrons à sa pratique politique et à son idéologie (ce qui ne veut pas dire que les autres mouvements religieux, ainsi que les aspects non politiques de leurs pratiques - à commencer par les aspects proprement religieux - soient dénués d'importance).

2 Après d'indispensables rappels historiques, l'on envisagera successivement les mécanismes idéologiques par lesquels le FNI propose une interprétation de la situation soudanaise et les voies et moyens de sa transformation, puis le réseau institutionnel formé par le Front et sur lequel celui-ci s'appuie, les actions qu'il mène et les oppositions qu'il suscite, pour finir par quelques considérations sur son possible devenir ${ }^{1}$. 


\section{Rappel historique}

3 C'est au début du XVI e siècle que le premier État islamique s'établit au Soudan : il s'agit du Royaume de Sennar, encore mal connu. L'islam soufi s'y répand, jouissant de la protection des souverains musulmans de ce royaume ${ }^{2}$, bien que les relations entre les cheikhs des confréries et ces derniers ne soient pas toujours excellentes. Autres acteurs de l'islamisation : les grands commerçants, dont les intérêts rencontrent en partie ceux du pouvoir et des chefs religieux. Ceux-ci, ainsi que l'armée, assurent en effet, par leur pouvoir de médiation pour les premiers et par la force pour la seconde, la sécurité des routes commerciales qui concourent en retour à la prospérité du royaume. Quant à la vie sociale, elle est empreinte, sous tous ses aspects, de religiosité d'inspiration confrérique, la mosquée constituant par ailleurs un lieu essentiel de sociabilité.

4 A l'époque du colonialisme de la Turqiyya (1824-1885), l'État se renforce et exerce un contrôle croissant sur l'économie (irrigation, lourde fiscalité), sur la société (écoles et tribunaux «modernes») et sur la sphère religieuse, soumettant les institutions islamiques à son autorité directe et leur apportant en retour des moyens financiers, ce qui les rend ainsi dépendantes de lui - en ville particulièrement - et en fait des « appareils idéologiques » dans le domaine politique.

5 Sous la période mahdiste (1885-1898), la mobilisation politique visant à mettre fin à l'oppression coloniale de la Turqiyya s'effectue sur des bases entièrement religieuses. Mais, à la mort du Mahdi, qui suit de peu la victoire de ses partisans, les problèmes liés aux divisions politiques intérieures et à la désorganisation de la production agricole s'intensifient et empêchent toute résistance sérieuse au nouveau colonialisme, anglais cette fois, qui, pourvu des armes les plus modernes, s'impose rapidement.

Durant la période coloniale anglaise, qui dure plus d'un demi-siècle (1898-1956), les systèmes économique et politique locaux se voient soumis, avec rigidité pour le premier et souplesse (accompagnée de recours occasionnels à la force) pour le second, à la logique de la production et de la reproduction capitaliste coloniale "classique ". Quant aux institutions religieuses, essentiellement confrériques, le pouvoir colonial fait usage à leur égard de diplomatie, tentant de se concilier - non sans résistance sporadique de leur part- leurs chefs religieux, en leur promettant ressources et place au sein du nouveau pouvoir en échange de leur loyauté. L'État colonial met en place de nouvelles institutions religieuses "officielles" auxquelles il rattache les institutions confrériques traditionnelles - cherchant à changer, par exemple, leurs méthodes d'enseignement -, lesquelles se trouvent, de ce fait, marginalisées matériellement et socialement et, à-tout le moins, «instrumentalisées». Sur le plan économique, par exemple, disciples et aspirants doivent participer à la production planifiée dans le cadre des grands projets agricoles - culture du coton essentiellement - souvent contre des salaires dérisoires, voire en échange de la baraka du cheikh de leur confrérie ${ }^{3}$.

Dans le Soudan indépendant, la période clé pour l'analyse de la transformation des rapports entre religion et pouvoir politique est celle de la présidence de Nimeiry (1969-1985) : celui-ci, privé du soutien de la plupart des catégories sociales ralliées sous la bannière des partis politiques d'opposition et/ou d'institutions confrériques envers lesquelles il nourrit la plus totale méfiance et qu'il prend pour cible de sa répression, tente de prendre appui sur les petites confréries soufies. Si son choix se porte sur ces dernières, c'est qu'elles se situent hors du champ de polarisation des deux «confréries" principales, celle des Ansar (qui n'est pas à strictement parler une 
confrérie) et celle de la Khatmiyya, et que, dénuées de capacité organisationnelle autonome forte, elles constituent des instruments de mobilisation faciles à manipuler et à utiliser idéologiquement ${ }^{4}$.

8 Durant les dernières années du régime de Nimeiry, après la réconciliation nationale entre le pouvoir et une partie des forces les plus notoires d'opposition au régime, à savoir le Parti Umma - émanation des Ansar - et les Frères musulmans, le régime entreprend de faire de ces forces des composantes intégrées à l'ensemble des appareils de l'État. Le pouvoir législatif, purement islamique selon Nimeiry et ses partisans, tend à faire de cet État autoritaire un "nouvel imamat ». L'on crée des tribunaux chargés de sanctionner tout comportement social jugé contraire à la chari'a et l'on constitue des comités d'Inspection des mœurs, appelés « comités pour le commandement du Bien et le pourchas du Mal». Les Frères musulmans sont au cœur de ce processus: leurs représentants comptent parmi les conseillers de Nimeiry. Dans de nombreux domaines; ils prennent en main les tribunaux de la chari'a et leurs dirigeants figurent au Conseil du Peuple (parlement) ; en outre, ils contrôlent les comités cités plus haut, ainsi que de nombreuses associations et organisations de Jeunesse, comme $l^{\prime}$ «Organisation de la jeunesse constructive».

Ils se constituent également une solide base économique en exerçant leur pouvoir sur la Banque islamique Faysal ainsi que sur ses succursales et ses diverses filiales opérant dans les secteurs du commerce et des assurances, et se livrant à diverses activités spéculatives. Quelques mois avant l'insurrection d'avril 1985, lorsque Nimeiry saisit la menace que représente pour lui la puissance des Frères, musulmans, il en fait arrêter et emprisonner un certain nombre. L'insurrection se déclenche alors qu'il se trouve aux États-Unis et que ces militants attendent son retour pour passer en jugement: les masses insurgées prennent d'assaut les prisons et libèrent tous les prisonniers politiques, Frères musulmans compris.

10 Sous le régime de Nimeiry, ceux-ci ont élargi leur base en intégrant de nombreuses autres associations musulmanes. Ce qui fait dire à Hassan al-Turabi, quand on l'interroge sur les Frères musulmans - dont il est devenu le dirigeant au milieu des années 60 - pour l'embarrasser à propos de leur participation au pouvoir de 1977 à 1985, «qu'il n'existe rien qui corresponde à ce que vous appelez les Frères musulmans». La seule organisation autorisée dans les dernières années de la présidence de Nimeiry est en effet l'Union socialiste. Le caractère clandestin de l'organisation des Frères musulmans ne permet d'ailleurs pas à Turabi d'en dire plus sur leur action. Toutefois, leur force se révèle clairement lors de la manifestation dite « du million » organisée en soutien aux « lois de septembre » de 1983, promulguées par Nimeiry et qualifiées par lui de " purement islamiques».

11 Notre objet n'est pas ici de faire l'historique des Frères musulmans, ni de décrire les formes d'organisation qui ont caractérisé les différentes étapes de leur histoire au Soudan jusqu'à l'apparition de l'actuel Front national islamique. Si nous évoquons ces principales étapes, c'est uniquement pour souligner l'importance des transformations intervenues dans les interactions entre conscience religieuse et système idéologicopolitique.

12 La source principale à laquelle ont puisé les Frères musulmans soudanais (tout comme les communistes soudanais, d'ailleurs), est l'Égypte des années 40 , dont les différents courants doctrinaux en matière religieuse ont influencé les étudiants soudanais vivant à l'époque au Caire. Revenus au Soudan, ces derniers ont diffusé, essentiellement 
auprès de leurs collègues, les doctrines qu'ils avaient assimilées en Égypte, alors que les membres du Parti communiste - le Front anti-impérialiste de l'époque - ont choisi, conformément à leur doctrine propre, d'investir le milieu ouvrier.

Les Frères musulmans se limitent étroitement au secteur étudiant jusqu'au milieu des années 60 , période où ils participent efficacement au renversement du premier régime militaire (1958-1964). Parce qu'ils sont désormais engagés dans le vaste champ de la confrontation politique, et compte tenu de la petite taille de leur formation, comparée à celle d'autres partis, ils créent un large front avec d'autres groupes religieux au recrutement étroit - comme les Ansar al-Sunna - gardant leur nom au sein de cette alliance baptisée « Front du pacte islamique », qui diffère de l'actuel FNI dans la mesure où les Frères se sont entièrement fondus dans ce dernier, devenu une entité unique dotée d'un seul conseil consultatif, de conseils de quartier avec bureau de propagande, d'un bureau pour les relations extérieures et la sécurité et, probablement aussi, de tout un appareil clandestin.

Grâce à la structure du FNI, les Frères musulmans retrouvent rapidement équilibre et activité après l'insurrection de 1985. En fait, alors que les partis traditionnels se sont enfoncés, durant les seize ans de pouvoir de Nimeiry, dans des problèmes les menant à la quasi-dislocation, le Front, lui, a pris racine et a même pu, de fait, participer au pouvoir dans les derniers temps du régime, participation qui n'a pas soulevé d'opposition sérieuse du fait de l'absence de forces politiques consistantes. Par ailleurs, le climat démocratique qui suit l'insurrection lui permet d'agir efficacement, comme le prouvent les résultats des élections législatives où le Front remporte la troisième place, après les deux partis traditionnels, l'Umma et l'Union démocratique (le Front obtient l'équivalent de la moitié des voix de l'Umma et des deux tiers de celles de l'Union). Ce qui est particulièrement significatif, c'est qu'il remporte la totalité des 28 sièges réservés aux diplômés de l'Université.

Si le Front a pu occuper d'emblée une telle position sur la scène politique, cela est bien sûr dû à sa capacité organisationnelle, à la fidélité de ses membres, au travail de persuasion de ses militants et à la diversité et à la solidité de ses sources de financement (évoquées plus haut, auxquelles s'ajoutent les cotisations et dons). Mais ce qui a "préparé le terrain» et accompagné son émergence, c'est sa capacité $\mathrm{d}$ '« idéologisation» de la conscience et du sentiment religieux. Les dirigeants du Front ont été à l'avant-garde de ceux qui allaient désormais traduire en termes religieux les problèmes économiques, sociaux et politiques du pays et réaliser leurs objectifs au détriment des partis traditionnels, dans un contexte de retournement de la conjoncture économique et de montée de la misère sociale qui allait faciliter cette " idéologisation » du religieux.

16 Le contexte, celui du milieu des années 80 , est celui des premières années de grande sécheresse. Elles ne font que révéler l'extrême fragilité économique d'un pays qui est en fait dans une situation désastreuse. Par ailleurs, malgré les menaces récurrentes d'éclatement politique (la première guerre civile a duré 17 ans et, en 1985, la seconde avait débuté depuis 2 ans), le Soudan forme une entité dont les parties sont socialement et économiquement interdépendantes, entité dominée sur tous les plans par un ensemble de populations qui se réclament dans leur majorité d'une origine araboislamique. Ces dernières sont principalement établies à Khartoum et dans les régions Centre et Est du pays, celles où se concentrent aussi les projets - peu nombreux - de développement, au détriment des régions du sud et de l'ouest. Dès le retournement 
économique du début des années 80 , la situation sociale du Soudan se reflète dans la vie quotidienne de la plupart des Soudanais, mis à part une petite partie d'entre eux - au nombre desquels figurent les militants et associés les plus en vue du FNI. Pour les autres, le lot quotidien, ce sont les problèmes de ravitaillement en produits de première nécessité, l'absence totale de médicaments, la difficulté d'accès aux soins et à l'instruction, l'augmentation vertigineuse du coût de la vie, l'aggravation du chômage et de l'insécurité (due à l'exode vers les villes provoqué par la sécheresse et la reprise de la guerre du Sud), ainsi que la dislocation de beaucoup de groupes familiaux. Toutes les catégories de population sont touchées, plus ou moins gravement, à l'exception des gros commerçants, hommes d'affaires, grands détenteurs d'exploitations agricoles mécanisées, hommes politiques et individus vivant d'activités parasitaires (change au noir, marché noir, contrebande, etc.). Ces derniers s'approvisionnent en abondance, accèdent aux soins médicaux dans les hôpitaux privés du pays ou à l'étranger, éduquent leurs enfants à grands frais (parfois payables en devises), se font construire de somptueuses villas et mènent un train de vie luxueux.

Contrairement à ce qui était encore le cas vingt ans auparavant, le Soudan des années 80 connaît une fracture sociale fortement marquée entre une petite minorité de familles très riches, vivant dans l'opulence, et une immense majorité de pauvres et de très pauvres luttant pour leur survie. Cette fracture se dessine tout particulièrement à Khartoum, où l'on ne peut échapper à la comparaison entre les quartiers de la haute société tels ceux de Ryad, Taef et Manchiyya et les quartiers les plus démunis aux baraquements de carton, de jute et de paille, où plus d'un million d'habitants vivent sans eau courante, sans électricité, sans moyens de transports réguliers, pour ne pas parler des services sanitaires et éducatifs.

Sans tomber dans des corrélations mécaniques, il est clair que, dans un tel contexte, des actions violentes de protestation risquent de se déclencher rapidement et que la prise de conscience de la situation peut s'accomplir et trouver son expression dans un cadre idéologique empruntant ses références et son langage à la sphère des représentations religieuses ou ethniques. Le FNI, de par sa structure interne et ses moyens humains et matériels, s'est trouvé en mesure de canaliser et d'orchestrer, dans le cadre de son idéologie particulière, l'expression de cette prise de conscience accomplie par un grand nombre de Soudanais. Ce qui, de plus, a beaucoup favorisé le FNI a été la faiblesse voire l'inanité - des autres organisations politiques, la détérioration du climat politique en général et l'instabilité socio-économique exacerbée par les catastrophes naturelles.

Ces mécanisme d'«idéologisation » - que nous traiterons plus loin - posaient deux questions principales : celle de la nature et de la place de la chari'a dans l'instauration d'un ordre social transformé, et celle de l'interprétation des désastres « naturels » et de leurs conséquences qui assaillaient la société soudanaise (sécheresse, désertification, pluies diluviennes de l'été 1988, famines, épidémies, appauvrissement, etc.). Les difficultés éprouvées par la conscience religieuse " populaire " pour trouver un sens à ces situations naturelles et sociales catastrophiques, dont la prise de conscience a été brutale, ont assuré au FNI une partie de son succès idéologique, par sa capacité à faire valoir comme la plus adéquate son interprétation de la situation et, sur la base de cette interprétation, à étayer son argumentation en faveur de l'application de la chari'a. Les mécanismes d'idéologisation grâce auxquels cette interprétation et cette argumentation ont prévalu, en canalisant l'expression de la prise de conscience des 
malheurs survenus et de la nécessité d'y apporter un certain type de remèdes, peuvent être analysés ainsi :

- une partie des aspects de la situation est détachée de son contexte et .présentée comme un tout ;

- ce tout est lui-même présenté dans l'absolu, en dehors de l'espace et du temps;

- les différents aspects de la situation sont abordés par fragments isolés et absolutisés, sans liens entre eux ;

- il est ainsi donné des phénomènes une image parfois déformée ou inversée par rapport à leur réalité ;

- un ensemble de représentations mêlant réalité et jugements de valeur sont élaborées, prétendant constituer une analyse de la situation et une doctrine en vue de sa transformation;

- cette doctrine est présentée comme devant constituer la solution radicale à tous les problèmes du pays ;

- elle est aussi présentée comme devant servir les intérêts de tous.

\section{Le FNI et les mécanismes d'idéologisation}

Nous exposerons sommairement dans cette partie le "processus d'idéologisation » dont le FNI fut l'acteur ; nous ne voulons nullement signifier par là que les activités du FNI se sont bornées à cette tâche ni que tous ses membres actifs s'y sont consacrés ; encore moins que les acteurs de ce processus visaient à "tromper le peuple » ou à déformer la vérité, alors qu'ils sont convaincus que ce qu'ils font est ce qu'il y a de plus juste et de plus opportun pour eux et pour les autres.

21 a) Lorsqu'il aborde le problème de la chari'a, le FNI en parle comme d'un équivalent de la religion et affirme que celui qui est contre son application n'est pas un musulman. En fait, en septembre 1983 comme en septembre 1988, il ne s'agissait de rien d'autre que d'appliquer les lois pénales relatives aux actions condamnées par décret divin (hudûd) et au châtiment légal. La chari'a en soi et dans sa totalité, en tant que système réglant l'existence d'un groupe social dans tous ses aspects et posant les fondements de la justice, fut laissée de côté. Certains membres du FNI ont relevé ce point et ont fait remarquer que la chari'a est davantage un ensemble de principes qu'un ensemble de lois, étant donné que, par nature, les lois sont partielles et qu'elles se réfèrent aux principes dont elles découlent. Or le FNI présente aujourd'hui les lois pénales de 1988 comme étant extraites - et non dérivées - de la chari'a. Il convient de noter que la Constitution soudanaise reste, quant à elle, essentiellement laïque ${ }^{5}$, et que les lois en question n'ont trait en réalité qu'à des questions de sécurité se posant du fait de l'insécurité croissante due à l'extension de la pauvreté, de la faim, de l'exode vers les villes - Khartoum en particulier - et du chômage. A parcourir la littérature du FNI, l'on croirait que la seule chose dont la société soudanaise est privée est la sécurité, et que la loi tirée de la chari'a doit rétablir l'ordre social en ramenant la sécurité. Quant aux causes réelles de l'insécurité, il n'en est nullement fait mention, sauf dans de rares cas et dans un autre contexte.

Le FNI établit un lien entre ce qui est considéré comme conséquences des catastrophes naturelles et la nécessité de l'instauration de la chari'a, en montrant que, comme le vol et l'adultère, la pauvreté, la faim et l'exode sont dus au non-respect des prescriptions divines. Les catastrophes naturelles elles-mêmes sont interprétées par certains 
membres influents du FNI comme une mise à l'épreuve, par Dieu, de la patience et de la foi des croyants et comme une occasion de retrouver le chemin qui mène vers Lui, le respect de la chari'a étant, pour ce faire, le meilleur guide. L'ensemble de ces interprétations et adjurations s'appuient sur des citations de versets du Coran et de textes de la Sunna, le plus souvent isolés de leur contexte, tant textuel qu'historique.

b) Tout en prenant ces parties de la religion pour le tout de celle-ci, le FNI en propose une image idéale et universelle, échappant aux contingences historiques, géographiques et sociales. Il réfère bien à ce qu'il appelle "L'État de Médine », mais là encore, sans mise en perspective historique de cet «État » et de la définition spécifique qu'on peut en donner. L'image proposée d'une société fidèle aux principes religieux est puisée dans un modèle a-historique. De même l'on cherche en vain une allusion aux événements et situations qui référeraient les rapports - changeants - entre pouvoir religieux et pouvoir politique dans les sociétés islamiques à des contextes historiques précis, depuis le jour de la sakifa - abri où se réunirent les émigrés (muhajirun), partisans (ansar) du Prophète à Médine -, jusqu'au règne des dynasties omeyyades et abbassides, en passant par les différents entre 'Ali et Mu'awiya. Ce mécanisme d'idéologisation (absolutisation d'une partie prise pour le tout, en vue de l'interprétation d'une situation présente et de l'assignation des fins et moyens permettant son amélioration ou sa transformation) aboutit à vider la religion de son contenu social et la société de son contenu historique. De celte manière, dans l'image de la société religieuse que le FNI érige en modèle, cette dernière se trouve exempte de tous antagonismes sociaux. Le "réservoir" où sont puisés les préceptes concrets permettant d'établir l'État islamique et de régir la vie individuelle et sociale quotidienne, à savoir les recueils de hadith, sont eux-mêmes coupés de leur contexte d'élaboration, les disputes jurisprudentielles et les différences d'opinions et d'interprétations étant occultées au profit de ce que Turabi a nommé la « jurisprudence de la nécessité ».

c) Ainsi, la réalité sociale du Soudan évoquée plus haut apparaît cloisonnée à travers un regard qui ne la perçoit que partiellement. On n'aborde au mieux que le problème de la diversité religieuse - et parfois ethnique - du pays, mais jamais la complexité des facteurs économiques, sociaux et culturels qui peut seule rendre compte de sa situation; la guerre civile qui le déchire sera le fait des fauteurs de troubles que sont, pêle-mêle, "chrétiens ", laïcistes et athées, tous infidèles qui mènent le combat contre l'islam tel que l'entend le FNI. L'image de la division sociale, du morcellement, n'est pas fondée sur la considération de l'opposition entre pauvreté et richesse ou entre groupes socioculturels, mais sur celle du clivage entre partisans sans réserve du FNI et opposants totaux (les "infidèles») ou partiels (divergeant d'avec lui sur des points déterminés, tel certains Frères musulmans qui refusent de renoncer au nom de leur groupe d'origine).

d) Le mécanisme d'inversion idéologique touche des concepts, réalités historiques, vérités considérées comme scientifiques ou mêmes événements quotidiens. L'on se contentera de quelques exemples :

- le concept de chûra (conseil, consultation) et celui de démocratie seront confondus. L'on peut admettre que "démocratie» signifie: liberté dont jouit la collectivité d'élire ses représentants et de leur reconnaître la compétence d'agir selon l'intérêt de la communauté, qui garde le droit de leur retirer sa confiance si elle le juge nécessaire; lois votées démocratiquement, s'appliquant de droit à tous, et pouvoir exécutif contraint de les faire 
appliquer. Le concept de chûra n'implique pas, quant à lui, l'existence de telles règles et obligations, mais seulement un avis consultatif que l'exécutif est libre de suivre ou non. Le FNI emploie ces deux concepts l'un pour l'autre et tourne ainsi la difficulté d'avoir à s'expliquer sur sa participation à une institution non-islamique telle que le parlement. D'autre part, il se présente comme un recours politique alternatif semblable aux autres, prônant le pluralisme politique, alors que ses orientations et beaucoup de ses initiatives tendent à écarter de toute participation au pouvoir les non-islamistes.

- A Omdurman, une famille chrétienne s'étant fait cambrioler par des voleurs ayant employé la manière forte pour lui soutirer argent et biens, les journaux proches ou dépendant du FNI ont rapporté l'incident comme une preuve de la nécessité d'appliquer la chari'a - ce qui n'est que logique dans leur optique - tout en affirmant que les chrétiens eux-mêmes réclamaient cette application ; or cette famille ne demandait que la protection des pouvoirs publics, ce qu'il est possible d'obtenir par divers moyens ${ }^{6}$.

26 e) II existe dans la littérature du FNI des allusions à la pauvreté, à l'ignorance, aux épidémies, à la guerre et aux souffrances des citoyens, mais pas aux causes véritables de cette situation. Pour le FNI, ce qui tient lieu d'explication, ce sont les complots internes et externes, l'indiscipline des citoyens, les séquelles du régime de Nimeiry que le Front a cependant appuyé durant la deuxième période - ainsi que la volonté de Dieu de mettre à l'épreuve les croyants. Le Front fait porter la responsabilité de la situation du pays à quelques individus ou groupes, nommés ou non, mais n'y voit pas la résultante d'un processus qui prend ses racines dans le mode de répartition très inégalitaire des ressources et du pouvoir et dans l'hégémonie économique, sociale et culturelle d'une minorité de citoyens.

f) Le FNI affirme qu'il ne pourra pas y avoir de progrès au Soudan ni de solution à ses problèmes économiques, sociaux, politiques et culturels si l'on n'y adopte pas les « lois de la chari'a ». Son argument principal est que l'Occident (à la fois chrétien et laïc) ne permettra jamais aux autres peuples de se développer de façon autonome. Par contre, la chari'a constitue le « choix culturel » de la société soudanaise par lequel elle pourra, grâce à une doctrine indépendante des idéologies occidentales, accéder au "développement autocentré ». Ainsi, selon un dirigeant du FNI, toutes les questions touchant à la répartition des richesses, à l'accès à l'éducation et aux soins, à la protection sociale et à la culture ont été suspendues dès l'Indépendance. La mise en place d'une législation pénale islamique constitue en fait le point de départ vers une transformation islamique totale de la société, qui reprendra «à zéro » tous ces problèmes éludés depuis l'Indépendance ${ }^{7}$. Mais le FNI n'en dit pas plus sur les objectifs concrets, ni sur les moyens, théoriques et pratiques, d'y parvenir. C'est qu'il s'agit uniquement de faire admettre : 1) que l'islam apporte une solution à tous les problèmes que connaît la société soudanaise ; 2) que les membres du FNI qui accéderont au pouvoir auront une moralité, une piété, une bonne volonté et des compétences qui leur permettront de s'attaquer victorieusement à tous ces problèmes ; 3) qu'une société réellement (ré)islamisée verra ces problèmes disparaître d'eux-mêmes. L'on n'enregistre cependant jusqu'ici aucune tentative de "traduction » des principes de l'islam (selon quelle interprétation?) en propositions de réforme constitutionnelle ou en programmes économiques, sociaux et culturels concrets.

g) Le Front se veut le vecteur du bien public, quelles que soient les appartenances sociales, ethniques et religieuses. Ainsi, si la chari'a doit être appliquée dans le « Nord musulman » du Soudan, les chrétiens y jouiront de la liberté de culte et d'association. 
Quant aux territoires du Sud, toutes les lois de la chari'a y seront appliquées à l'exception des hudûd, dont la définition sera conforme à la chari'a, mais non le châtiment : l'adultère, par exemple, sera prouvé selon les normes de la chari'a, alors que le châtiment encouru sera une peine de prison ${ }^{8}$. Ceux qui s'opposeront à l'application de la chari'a seront traités soit en comploteurs (pour ce qui est des laïcistes et des athées) soit en ignorants (pour les chrétiens, qui ne savent pas où réside leur intérêt, et les musulmans qui ne comprennent pas la nécessité d'appliquer la juste loi, telle qu'elle est définie par le Front). La notion de «bien public» semble cependant souvent malmenée par certains membres du FNI, qui reconduisent, dans leurs pratiques au sein des institutions qu'ils dominent, celles qu'ils ont dénoncées et qui fleurissaient dans les partis «traditionnels»: clientélisme, favoritisme, etc. Il est certain que dans les secteurs que contrôle le FNI, le recrutement professionnel s'est souvent limité à sa clientèle. Au cours des derniers mois, le secteur public a vu beaucoup de ses directeurs exécutifs licenciés pour être remplacés par des éléments du Front. De même, les banques islamiques - en particulier la Banque Faysal - et leurs filiales emploient essentiellement des membres du FNI. Quant à leurs dirigeants, ils mènent des opérations financières et d'investissement lucratives, à caractère souvent spéculatif ; les « retombées » en sont visibles : transformation de leur train de vie, devenu dans ces dernières années des plus luxueux.

Le travail d'« idéologisation » ne suffit cependant pas, on t'a dit, à définir les activités du FNI. En tant que réalité proche d'un parti politique, et pour mener à bien son projet de transformation de la société soudanaise selon ses valeurs et interprétations, il lui a été nécessaire de créer des institutions et de s'appuyer sur d'autres, déjà existantes.

\section{Le FNI et ses institutions}

La «base " économique du FNI est, on l'a vu, constituée de banques et sociétés islamiques qui effectuent de nombreuses opérations financières et commerciales à travers le pays.

Sur le plan social, le FNI a créé des organisations de jeunesse comme «La jeunesse constructive " et «Les pionnières de la Renaissance " qui, soutenues par des institutions économiques et politiques, mènent des actions sociales précises, telle la réduction des frais de mariage, en organisant ce qu'elles appellent "mariage d'alZahra' », sorte de cérémonie collective. Le Front, par ailleurs, a participé à la distribution d'aide aux sinistrés à la suite des inondations de l'été 1988; il a, de même, déployé son activité auprès des réfugiés et déplacés musulmans. En outre, la présence de membres du Front est notoire - voir hégémonique - au sein de l'Agence islamique d'aide et autres institutions islamiques dont le siège est à Khartoum, par exemple le Centre islamique africain, qui s'occupe de l'éducation et de la formation des musulmans d'Afrique.

Sur le plan politique, les membres du Front poursuivent une intense activité militante, surtout en milieu étudiant, où ils ont contrôlé pendant plusieurs années les Unions d'étudiants de l'Université de Khartoum, de la branche soudanaise de l'Université du Caire et de l'Université islamique d'Omdurman. Ces Unions, ainsi que les syndicats professionnels conquis par le Front, sont considérées comme les principales pépinières où peuvent se révéler et se former de futurs chefs politiques. Par ailleurs, on le sait, le Front occupe la troisième place parmi les partis politiques représentés à l'Assemblée 
Constituante (Parlement), mais de par ses capacités d'organisation et les effectifs de ses membres actifs, il dépasse de beaucoup ce que cette troisième place pourrait laisser supposer. Il détient des postes ministériels importants : Vice premier ministre, Affaires étrangères, Commerce intérieur, Affaires sociales et Aumône légale (zakât), ainsi que le poste de procureur général et plusieurs postes de gouverneurs et gouverneurs-adjoints. Sur le plan culturel, le Front possède un secteur d'information actif soutenu par d'importants moyens financiers et techniques et dirigé par des cadres très qualifiés (dont beaucoup ont été formés dans des universités et institutions américaines). Ce secteur dispose notamment d'une presse quotidienne ou bi-hebdomadaire (al-Râyât, Alwân, etc.) et hebdomadaire ou mensuelle (Sanâbil, Afkâr); il compte également plusieurs maisons d'édition. Par ailleurs, le Front a constitué une communauté d'artistes regroupés sous le nom de Namârek, couvrant les domaines du chant, du théâtre et des arts plastiques. Des membres du Front exercent aussi une grande influence au sein de ce que l'on nomme "L'Union des hommes de lettres soudanais", rivale de "L'Union des écrivains soudanais ». Toutes ces institutions (ou les membres du Front adhérents de celles-ci), quoique autonomes, sont en liaison étroite, et sont clairement les porte-parole du FNI dans les différents champs de la pratique sociale.

Sur la base de son idéologie, assez largement partagée par certaines catégories de la population, et grâce à ses institutions spécialisées et à la présence de ses membres dans d'autres institutions, le FNI a pu mener un certain nombre d'actions qu'il reste à examiner ci-après.

\section{L'action du FNI}

Le FNI est intervenu à la fois par des actions sociales concrètes et par des opérations proprement politiques. Lors des catastrophes naturelles, il a fourni d'importants secours (médicaments, tentes, distribution d'allocations en numéraire, etc.). Il est passé, pour mener à bien ces actions, par l'intermédiaire de ses organisations de jeunesse et par les institutions d'aide islamique, dont l'efficacité a augmenté sa popularité. Il accompagnait ce travail d'assistance de campagnes d'explication des causes des catastrophes qui éveillaient, dans la population secourue, enthousiasme et frayeur tout à la fois.

Dansée domaine proprement politique, le FNI a organisé plusieurs campagnes de pression - voire de menaces directes - . et/ou de protestation contre des parlementaires et autres opposants hostiles à son action: manifestations autour du siège de l'Assemblée constituante; menace contre un député particulièrement hostile aux nouvelles lois pénales, de faire, le premier, l'expérience des châtiments qu'elles prescrivent contre les " comploteurs " ${ }^{9}$; déclaration d'un dirigeant du FNI relative à ses milices armées, «dont il vaut mieux éviter l'intervention ${ }^{10}$; publication, dans la presse islamiste, de "listes d'honneur », sur lesquelles figurent les sympathisants du Front, et de blâmes contre ses opposants, considérés comme ennemis de l'islam, avec ce que cette désignation suppose comme risques de châtiments; menaces adressées à des étudiants manifestant leur opposition au projet de nouvelles lois pénales (de la chari'a) d'avoir à se repentir publiquement, etc.

Pendant tout le temps qu'a duré le débat autour de ce projet, cependant, les membres du Front n'ont soulevé publiquement aucune question précise, d'ordre moral, jurisprudentiel ou religieux relativement à ce sujet, ce qui ne pouvait que les priver de 
l'appui de certaines catégories «influentes » de la population: intellectuels, juristes... Ils ont gardé un silence total sur la question du statut de la femme (port du voile, mixité à l'Université). Même silence sur ce qui les oppose depuis les années 60 aux confréries, aux partisans de la Sunna, voire aux Frères musulmans qui revendiquent l'identité propre de leur mouvement. Ces divergences n'ont été évoquées que dans des réunions internes.

Sur le plan de la tactique politique, le Front tente souvent de diviser les groupements qu'il ne contrôle pas ou qui lui sont clairement opposés. Une grande partie des syndicats d'ouvriers, d'employés et de professions libérales échappent totalement à son influence. L'Union générale des ouvriers du Soudan jouit d'une grande indépendance vis-à-vis des partis, quelle que soit leur orientation, alors que les syndicats des avocats, médecins, ingénieurs et professeurs de l'Université de Khartoum sont considérés comme globalement « de gauche » au sens large. Or, on a vu apparaitre au sein de puis contre - ces syndicats, légalement reconnus et dont les instances sont élues démocratiquement, des tendances qui se constituent en associations parallèles et rivales et qui se disent " patriotes " : l'on a ainsi un Syndicat des avocats patriotes, une Association des médecins patriotes, un Regroupement syndical national, etc., dont le rôle est de laminer les rangs des syndicats reconnus au cas où ils s'opposeraient aux législations dites - par le FNI - «islamiques ", ou à l'orientation du Front ou du gouvernement. Toutefois, ces nouvelles formations restent très fragiles et n'ont pu s'opposer aux mouvements émanant des syndicats reconnus quant à la protection des libertés syndicales. Lors de la grève des médecins de février 1989, par exemple, l'Association des médecins patriotes a publié un communiqué dans lequel elle exprimait son soutien aux revendications des médecins, mais a condamné la grève, qui avait pourtant été suivie à $100 \%$ dès son déclenchement.

Sur le plan extérieur, le Front tente d'entrer en relation avec des milieux qui, traditionnellement, n'étaient liés à aucun groupe ou parti ; il cherche, par ce biais, à consolider sa position intérieure et à contrer l'hostilité dont il est l'objet de la part de nombreux gouvernements de pays arabes et islamiques. Ainsi a-t-il pris contact avec le pouvoir pakistanais peu de temps avant la mort de Zia UI-Haqq, qui cherchait lui aussi à imposer l'application de la chari'a dans son pays. Les rencontres entre des dirigeants du Front et des organisations islamiques pakistanaises se sont intensifiées ; on a eu aussi recours à ces dernières lors de l'organisation de conférences de soutien à l'application de la chari'a organisées au Soudan. Après la mort de Zia Ul-Haqq, les contacts du Front, entamés plusieurs années auparavant avec des organisations islamistes tunisiennes, se sont multipliés ${ }^{11}$. Le Front est également entré en relation avec le Parti communiste chinois (allant jusqu'à l'échange de visites cordiales) et avec la Corée du Sud et le Japon. Il est à remarquer qu'aucun lien ne semble exister entre le Front et des organisations européennes et américaines, bien que l'Europe - la Grande-Bretagne en particulier et les États-Unis aient été les lieux d'élection pour leur formation académique mais aussi leurs activités commerciales et leur soins médicaux, de nombreux dirigeants du FNI. stratégies déployées, le Front rencontre une vive résistance parmi diverses catégories sociales, et ceci pour de nombreuses raisons. 


\section{Les oppositions au Front national islamique}

41 Opposition tacite ou désaveu, d'une part, lorsque ces catégories se mobilisent pour protester ou revendiquer sur des thèmes qui ne rejoignent pas - voire contredisent ceux du FNI. En 1988, alors que les débats s'étaient à nouveau engagés sur la question des nouvelles lois pénales "islamiques", en quelques mois, plus de vingt grèves d'ouvriers, d'employés et de cadres ont éclaté, en protestation contre la détérioration de leurs conditions de vie, grèves au cours desquelles des slogans anti-FNI furent largement repris. En juillet 1988, dans un communiqué commun rédigé par 22 syndicats (ceux des agriculteurs, des professeurs de l'Université de Khartoum, des employés de banques, de l'eau et de l'électricité, etc.) et lors d'une réunion de la Coordination des syndicats, le rejet des directives du Front a été unanimement proclamé. D'autre part, le Syndicat des avocats et l'Association des droits de l'homme ont organisé des rencontres, conférences et meetings où l'on expliquait les problèmes constitutionnels que ne manquerait pas de faire apparaître la législation proposée par le FNI, ainsi que les conséquences qu'elle aurait sur l'unité du Soudan. Intervenant essentiellement par le bais d'articles de presse, puis par des contacts pris à l'étranger visant à déclencher des pressions internationales sur le Soudan, le Conseil soudanais des Eglises a joué un rôle important pour montrer comment les projets de loi touchant aux croyances religieuses pouvaient semer la division parmi les citoyens ${ }^{12}$.

En décembre 1988, la réduction du niveau de vie consécutive à la montée considérable des prix des denrées de première nécessité a poussé nombre de Soudanais à participer à d'importantes manifestations, prenant d'assaut, à Khartoum, le siège du Conseil des ministres et, dans les provinces, manquant de tourner à la désobéissance civile si le gouvernement n'était pas revenu sur certaines mesures d'augmentation des prix. Bien que dirigées d'abord contre le gouvernement de l'époque, il est clair que de telles actions collectives constituaient un désaveu implicite des mots d'ordre du Front proclamant: "L'islam avant le pain !» ou «Vite, la chari'a ou nous mourrons !», passant outre à la situation de grave pénurie que connaissait le pays. Or, c'était bien pour tenter d'assurer leur pain et celui de leurs enfants que des millions de Soudanais descendaient dans la rue; bien plus, des partisans et sympathisants du FNI défilaient avec eux.

Maints écrivains et journalistes musulmans soudanais ont écrit sur les aspects contradictoires de l'idéologie du Front. D'après certains, la loi pénale impliquait nécessairement l'existence d'une constitution islamique préalable, d'un régime islamique dirigé par un imam légitime, mais aussi d'un État qui assurerait à la population le minimum de subsistance avant de veiller au respect des préceptes divins ${ }^{13}$. D'autres ont insisté sur le fait qu'avant d'être châtiment, l'islam est d'abord pédagogie. D'autres encore ont souligné la contradiction existant dans le fait de revendiquer l'application de la chari'a au moyen d'institutions non-islamiques ${ }^{14}$. On a soulevé le problème des concepts de la jurisprudence liée à la chari'a et celui de la définition des actions condamnées par statut divin (l'abjuration et l'adultère méritentils le châtiment légal, dans quelles circonstances et que doit-il être ?) ${ }^{15}$. On s'est posé des questions sur l'histoire islamique (a-t-elle jamais connu l'application de ce que prône le Front islamique ?). L'on s'est interrogé sur l'école (madhhab) - ou les écoles dont s'inspire le Front dans ses positions juridiques. Quelques-uns sont même allés jusqu'à accuser Hassan al-Turabi de blasphème pour certaines de ses opinions 
religieuses. L'on n'a pas manqué de relever ce qui apparaissait comme une contradiction entre l'hostilité à la laïcité, manifestée par le Front, et l'influence de la culture occidentale qui apparaît dans la conception qu'a le Front de l'activité politique. Turabi, principal dirigeant et penseur du Front, a en effet poursuivi ses études en France et en Angleterre, et certains commentateurs en ont conclu que les fondements de l'idéologie du Front pourraient être beaucoup plus séculiers qu'il n'y paraît.

Le Front a prétendu que les propositions de «lois islamiques » qu'il présenterait au Conseil des ministres et à l'Assemblée avaient été rédigées dans le cabinet du procureur général et avaient reçu l'aval des ulémas et des universitaires compétents. Or, il a été récemment prouvé que ces propositions ne représentaient que les options du Front et qu'elles avaient été rédigées par un petit groupe de ses spécialistes, sans avoir été soumises à l'approbation d'ulémas ou d'universitaires extérieurs au Front. Le directeur du département d'études spécialisées dans la chari'a à l'Université de Khartoum a déclaré qu'il n'avait pas vu le projet ni été consulté à son sujet ${ }^{16}$. Il a été également prouvé que les groupes parlementaires des partis n'ont pas davantage discuté de ces propositions. A l'intérieur de l'Assemblée, les leaders de l'Union démocratique (qui participent, avec le FNI, au pouvoir) ont organisé l'opposition à ces propositions et réussi à les faire considérer, par la commission législative du Parlement, comme l'un des cinq projets de lois alternatifs candidats au remplacement des lois de septembre 1983. L'opposition avançait que la loi proposée n'avait été élaborée que par le Front, alors que les propositions concurrentes l'avaient été avec la participation d'éléments divers et qu'en outre, elles soulignaient les droits fondamentaux mentionnés dans la Constitution comme les droits de l'homme, la liberté religieuse, etc. Début 1989, la commission législative de l'Assemblée examinait toujours les propositions de loi en question et elles ne semblaient pas devoir être ratifiées, étant donné, d'une part, l'existence de diverses formes de pression et, d'autre part, l'apparition de complications liées aux conditions posées pour l'arrêt des combats dans le Sud (une conférence de paix se déroulant dans un contexte conforme à la Constitution, et non dans celui de l'instauration de « lois islamiques »). Enfin, des pressions internationales ont plongé le Soudan dans un isolement qu'il n'avait connu qu'aux derniers jours de Nimeiry.

Une part de l'opposition au FNI s'explique aussi simplement par le fait que ce dernier a maintenant fait "officiellement " l'expérience du pouvoir, et qu'ainsi ses leaders ont été jugés par les différents groupes sociaux sur ce qui apparaissait comme «leurs actes ». Il a par exemple été dit ou écrit, sur la foi de cette expérience, que tout musulman, même bien intentionné, ne réussit pas nécessairement à atteindre les buts justes qu'il s'était fixé ; que, réciproquement, tout homme politique qui, au service de nobles idéaux, s'acquitte avec succès de sa tâche, n'est pas nécessairement un promoteur de l'islam; enfin, que tout homme politique islamiste n'est pas nécessairement dépourvu d'ambitions personnelles et séculières et qu'il peut essayer de les réaliser en ayant recours à des moyens qu'islamistes et non-islamistes s'accorderaient à trouver immoraux. Ainsi certains membres du Front ont-ils été soupçonnés de s'être approprié des biens publics et de les avoir utilisés dans leur intérêt propre, d'avoir participé à des opérations frauduleuses ou de pratiquer le favoritisme (au cours de ces derniers mois, des procédures qui les concernaient, relatives à des opérations de corruption bancaire ${ }^{17}$, ont été interrompues). 

peser lourdement sur le devenir de cette formation, sur lequel on s'interrogera pour finir.

\section{Le devenir du FNI}

Vers le milieu des années 70, les symptômes de divergence se sont multipliés entre les anciens Frères musulmans, tenant de la formation pédagogique et de l'engagement moral et les partisans, en leur sein, d'une nouvelle formation orientée politiquement ; l'on a ainsi abouti à une scission : un groupe réduit à un « groupuscule » a gardé le nom de Frères musulmans, tandis qu'un second groupe élargissait sa structure pour se fondre dans le Front national islamique.

Le Front a cependant assez vite vu partir nombre de ses membres, qui exprimaient de cette manière leur désaccord avec sa politique indulgente et son silence à l'égard de questions relatives notamment aux activités mixtes, mettant en présence jeunes gens et jeunes filles au cours d'excursions, de rencontres de réflexion politique et d'activités artistiques comme le théâtre, le chant et autres loisirs. Par la suite, à l'intérieur du Conseil consultatif, la plus haute instance du Front, de profondes divergences ont éclaté entre ceux qui refusaient de participer au gouvernement avant la ratification des lois islamiques, et ceux qui acceptaient d'y rentrer immédiatement, alléguant du fait que leur présence au sein du pouvoir les rendrait mieux à même de faire passer ces lois. Finalement, il a été entendu que l'on participerait au pouvoir à condition que les lois en question soient ratifiées dans une période limite de deux mois, à l'issue desquels on démissionnerait si elles ne l'étaient pas. Plus de six mois après, aucune de ces deux propositions ne s'était réalisée.

Par ailleurs, l'ébauche d'une nouvelle orientation apparaît au sein du Front parmi ses hauts responsables les plus récents, qui ne cachent pas leur gêne devant ce qu'ils considèrent comme une étroitesse de vues de la part de leur organisation, et qui témoignent volontiers d'une grande tolérance à l'égard des idées " modernistes » des autres groupes politiques, y compris les communistes, cela dans le but d'étendre et de consolider la doctrine islamique. Quelques-uns ont commencé à blâmer clairement certaines pratiques des membres du Front appartenant au pouvoir et leurs critiques se sont amplifiées au lendemain des catastrophes naturelles, lorsque s'est creusé encore plus profondément - et s'est mieux révélé - le fossé existant entre riches et pauvres, avec ce que cela implique de risques d'instabilité au niveau social et politique ${ }^{18}$.

Quelle que soit la cohérence des thèses idéologiques du Front, de tels conflits et tendances nouvelles étaient à prévoir, étant donné l'importance de l'organisation, ses réseaux de relations, sa dynamique, la diversité sociale de son recrutement et, de ce tait même, l'apparition de grandes différences, sur les plans matériel et social, entre les dirigeants et la «base». Les premiers, pour beaucoup, ont le train de vie - simple constat sociologique dépourvu de jugement moral - de la peu nombreuse mais très «visible» nouvelle bourgeoisie soudanaise, dont ils font partie, alors qu'il ne semble pas que la base diffère, par ses modes de vie et moyens d'existence, du reste de la société. L'idéologie du Front a beau gommer en partie ces différences en en atténuant la portée, elles ne peuvent, aux yeux de ses membres et sympathisants comme à ceux des Soudanais qui lui sont extérieurs, passer totalement inaperçues. En outre, il semble que 
des clivages internes de génération apparaissent dans le FNI entre jeunes militants et dirigeants plus âgés, dont certains représentent la « vieille garde ».

Des possibilités de scission apparaissent dans deux directions : d'une part, vers plus d'ouverture et de tolérance intellectuelle et sociale : d'autre part, vers la formation de groupes radicaux tant sur le plan de la pensée que de l'action politique ; ici les chances d'apparition d'extrémisme » et d'intolérance sont grandes et le terrain est propice à la violence. Des rumeurs courent sur l'existence d'une organisation "secrète » ayant reçu un entraînement spécial et sur des armes dont le Front serait en possession. Ce qui est maintenant sûr, c'est que des incidents sanglants ont eu lieu à l'Université de Khartoum, des groupes de militants étudiants du Front ayant attaqué d'autres étudiants qui assistaient à une pièce de théâtre ayant pour thème la chute de la Bastille et qui se donnait au Club des professeurs de l'Université, attaque menée sous prétexte que le spectacle était « contraire aux bonnes mœurs ». Des militants du Front, armés de chaînes et de couteaux, ont également assailli le local d'un journal opposé à leur organisation et l'ont mis à sac ${ }^{19}$. Enfin, on a pu voir récemment des manifestations du Front encadrées par un service d'ordre spécialisé dans la défense «musclée». Ces phénomènes doivent être notés sans en conclure pour autant à un glissement global de la formation étudiée vers le recours à la violence politique.

L'étude que l'on vient de lire a appréhendé le FNI comme un tout, à quelques nuances près. Il serait cependant indispensable d'aller plus loin et de procéder à des études plus détaillées des divers niveaux organisationnels du Front, de la nature et de la diversité de ses actions, de l'origine sociale de ses membres, de leur formation, de leurs trajectoires sociales et de leurs systèmes de valeurs.

\section{NOTES}

1. Cet article a été écrit en janvier 1989, avant que le Front national islamique, ayant participé au pouvoir depuis les élections libres de 1986, n'en ait démissionné.

2. En ce qui concerne les conditions de la propagation de l'islam soufi au Soudan, voir l'introduction de l'ouvrage de Muhammad al-Nur Ben Daif Allah. At-Tabaqat. KUP, Khartoum. 1985 ( $3^{\mathrm{e}}$ éd.).

3. Ce système existait dans les périmètres irrigués par pompes dans la province du Nil Blanc, où la communauté des Ansars jouissait d'une grande influence. Les disciples effectuaient tout le travail agricole, en échange de quoi ils ne touchaient que ce qui pouvait leur permettre d'assurer leur subsistance, avec l'espoir d'être récompenses au jour du Jugement Dernier. Le même système était en vigueur dans la région Nord du Soudan, sous le contrôle de la confrérie de la Khatmiyya ; alors que le coton constituait la principale culture des périmètres du Nil Blanc, c'est dans les vergers que les disciples de la Khatmiyya travaillaient au Nord.

4. Voir dans le Ph.D. de l'auteur, On ideology: the case of religion in Northern Sudan, University of Connecticut. USA, 1980, la partie consacrée à la « récupération » des confréries par Nimeiry et en particulier des confréries de la Badrabiyya (branche de la Qadiriyya) et de la Samaniyya (d'origine locale). 
5. L'opposition religieuse au projet de nouvelles lofs pénales est essentiellement venue des Jumhuriyyin (Frères républicains), ennemis traditionnels du Front, puis des Frères musulmans "maintenus", qui ont souvent allégué que les orientations de Turabi représentaient une déviation par rapport aux principes fondamentaux de la religion selon al-Hanati. Les Frères républicains, eux, rappellent que le principe même de retenir la chari'a comme fondement possible des lois n'a jamais été soumis au vote (déclaration de l'avocate Asma Mahmud Muhammad Taha au cours d'une conférence au syndicat des avocats le 19/9/1988). Quant aux Frères musulmans, ils basent aussi leur opposition sur le fait que le chari'a est indivisible et ne peut s'appliquer dans certaines régions du pays et non dans d'autres.

6. Alwan, 4/10/1988.

7. D'après les textes d'une conférence tenue à l'Université de Khartoum le 20/9/1988, durant laquelle ont pris la parole divers dirigeants du Front dont Mahdi Ibrahim (cf. al-Usbu du 22/10/1988).

8. Discours de l'avocat Taha Ibrahim lors de la conférence des avocats citée en note 5.

9. Menaces dirigées contre le député Mansur al-Ajab, cf. al-Ayyam du 23/9/1988.

10. Al-Raya du 23/9/1988.

11. Le Front entretient des relations étroites avec l'Iran, dont témoignent des visites répétées de dirigeants du FNI dans ce pays, ainsi que l'éloge des réalisations islamiques de la Révolution iranienne. Ces liens se sont concrétisés dans l'attitude adoptée par le Front par rapport à la guerre Iran-Iraq, durant laquelle il n'a jamais pris position en faveur de ce dernier. Les relations avec les mouvements islamistes tunisiens sont également régulières, surtout depuis que des divergences ont opposé le FNI aux Frères musulmans égyptiens. Des membres du Front ont par exemple rencontré Ghannuchi. Par contre, le FNI ne semble pas avoir de relations significatives avec l'Arabie Saoudite, au niveau organisationnel du moins.

12. Sudan Times du 15/7/1988.

13. L'une des personnalités les plus notoires à soutenir cette opinion est l'ancien président de la magistrature, 'Uthman al-Tayyeb, qui a participé à un débat télévisé avec le Dr. Hassan al-Turabi, à qui il apportait la contradiction.

14. Cf. par exemple ce qu'a écrit Abu Dafira (chef d'un des groupes religieux de l'Université de Khartoum) à propos de la démocratie et de l'Assemblée constituante, qu'il a qualifiée de " peste » dans son livre 'ilm an-sa'a bil raqm 13 wa naqd al-dawla al-dimuqratiyya, Khartoum (sans mention d'éditeur ni date).

15. Cette objection a été avancée par Sadiq al-Mahdi, chef des Ansar et Premier ministre, lorsque le projet de nouvelle loi pénale a été soumise au Conseil des ministres.

16. Al-Raya du 2/10/1988. En ce qui concerne le projet de loi comme représentant le seul avis du FNI. cf. Al-Ayyam du 18/7/1988, la rubrique de Bachir Muhammad Saîd.

17. Certains dirigeants du FNI ont été accusés d'avoir mis la main sur des terrains de l'État sans aucun droit, et d'autres délits de détournement de biens publics (cf. al-Watan, articles de novembre 1988 et avril 1989).

18. Voir le premier numéro du journal du FNI, al-Sanâbil.

19. C'est al-Watan qui a publié avec assiduité ces informations. 
INDEX

Mots-clés : Soudan

\section{AUTEUR}

IDRIS SALIM ELHASSAN

Université de Khartoum 